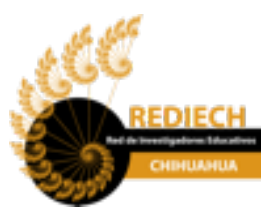

Red de Investigadores Educativos Chihuahua A.C. Chihuahua, México www.rediech.org

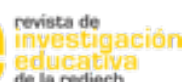

de la rediech

ISSN: 2007-4336

ISSN-e: 2448-8550

http://www.rediech.org/ojs/2017/index.php/ie rie rediech/index

Jesús Adolfo Trujillo Holguín

2019

\title{
[Editorial]
}

\section{INVESTIGACIÓN CIENTÍFICA, ACCESO ABIERTO Y DEMOCRATIZACIÓN DEL CONOCIMIENTO}

IE Revista de Investigación Educativa de la REDIECH, 10(19), pp. 5-10.

DOI: http://dx.doi.org/10.33010/ie_rie_rediech.v10i19.770

\section{(c) (1) (9)}

Esta obra está bajo licencia internacional

Creative Commons Reconocimiento-NoComercial 4.0.

CC BY-NC 4.0 


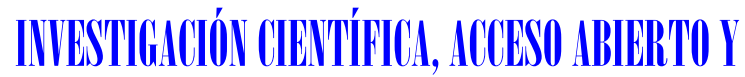

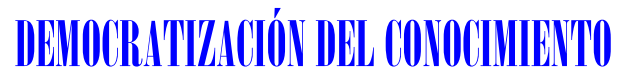

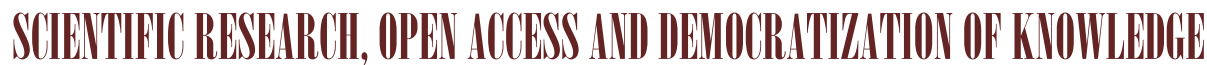

TRUJILLO HOLGUÍN Jesús Adolfo ${ }^{1}$

lirector

DOI: http://dx.doi.org/10.33010/ie_rie_rediech.v10i19.770

La investigación científica debe estar orientada hacia la resolución de problemas sociales. Diversos autores coinciden en señalar que esta actividad tiene como propósito esencial la generación de conocimientos que proporcionen bases para el desarrollo del país y a la vez contribuyan a la generación de políticas públicas adecuadas (López, Sañudo y Maggi, 2013). La Ley de Ciencia y Tecnología establece que la política del Estado en la materia tiene como propósito: "Incrementar la capacidad científica, tecnológica, de innovación y la formación de investigadores y tecnólogos para resolver problemas nacionales fundamentales, que contribuyan al desarrollo del país y a elevar el bienestar de la población en todos sus aspectos" (Gobierno de la República, 2002, p. 65).

El acceso abierto al conocimiento científico se concibe como un bien común y un derecho universal, máxime si se trata de trabajos derivados de proyectos y actividades investigativas financiadas con recursos públicos. Esta situación de alguna manera se contrapone a las tendencias mundiales en materia de circulación y uso del conocimiento científico por parte de las revistas de paga y de los grandes repositorios comerciales (Scopus y Web of Science), quienes condicionan el acceso a la información a cambio de cuotas individuales o institucionales para la descarga de contendidos.

La democratización del conocimiento supone que los resultados de las actividades de investigación científica estén al alcance de todos. En México ya existen atisbos de que comienza a cuestionarse las políticas que impulsa el Consejo Nacional de Ciencia y Tecnología (Conacyt) en materia de acceso abierto, aunque todavía queda pendiente una transformación de fondo en los criterios de evaluación de la calidad de la producción científica que actualmente se basan en el factor de impacto y el lugar donde están indizadas las revistas (Scopus y Web of Science). Igualmente se

${ }^{1}$ Universidad Autónoma de Chihuahua, México. Correo electrónico: jatrujillo@uach.mx. ID: http://orcid.org/0000-0002-6738-1878. 
requieren estrategias de apoyo a las iniciativas encaminadas a favorecer el acceso abierto y el fortalecimiento técnico y tecnológico de las publicaciones que cumplen con ciertos estándares de calidad.

A nivel internacional -y particularmente en Europa- surgió la iniciativa conocida como el Plan S, que busca garantizar que todos los trabajos subvencionados con recursos públicos estén disponibles en revistas y plataformas de acceso abierto a partir del 1 de enero de 2020. Esta medida no convence del todo a los especialistas en el tema debido a que transfiere la responsabilidad del pago por lectura (suscripciones) al pago por publicación, aun y cuando los costos sean asumidos por las instancias que patrocinan la investigación o por las instituciones de adscripción de los propios investigadores. Se exploran varias alternativas para hacer frente a los desafíos para la democratización del conocimiento, como es la vía verde (autoarchivo de trabajos en repositorios institucionales), vía dorada (publicación en revistas de acceso abierto) y vía diamante (sin pagos por suscripción ni por publicación) (Ministerio de Economía y Empresa, 2017).

En nuestro país se están dando pasos importantes en el tema y en 2019 ocurrieron dos eventos importantes a destacar. El primero se refiere a la promulgación de la decimoprimera reforma al artículo tercero constitucional que consagró el derecho para que toda persona pueda "gozar de los beneficios del desarrollo de la ciencia y la innovación tecnológica" (p. 4) y además compromete al Estado para que garantice el acceso abierto a la información que se derive de estas actividades. Este cambio normativo trajo como consecuencia que actualmente esté en discusión una nueva Ley General de Ciencia y Tecnología, situación que ha propiciado la apertura de foros de discusión donde el propio Conacyt está replanteando sus políticas de financiamiento en el sector.

El segundo acontecimiento se dio en un contexto más amplio y de cierta forma viene a consolidar las iniciativas que existen en la región latinoamericana desde hace más de 30 años. El Sistema de Información Científica Redalyc lanzó el proyecto R2020: Modelo de publicación abierto, propiedad de la academia, sin fines de lucro, sustentable, con métricas responsables y no subordinado que busca fortalecer el trabajo que la Red de Revistas Científicas de América Latina y El Caribe, España y Portugal impulsa para promover una política de acceso abierto sin costos por publicación o procesamiento (APC). El proyecto reconoce que -a diferencia de lo que ocurre por ejemplo en Europa- en América Latina mayoritariamente la investigación se financia con recursos públicos y sería incongruente que los resultados que derive sean exclusivos para grupos particulares o empresas que se beneficien económicamente de tales actividades (Aguado-López y Becerril-García, 2019).

Desde la Red de Investigadores Educativos Chihuahua vemos con agrado que se esté discutiendo el tema del acceso abierto, y sobre todo que se consoliden estrategias como las que impulsa Redalyc, pues suponen valorar la producción científica por su calidad y no solamente por el lugar donde se encuentran indizadas. Igualmente resulta valioso el acompañamiento y capacitación que brindan para fortalecer los procesos editoriales que llevan a transitar de la publicación electrónica a la digital. Revistas como la nuestra disponen de una plataforma para la conversión de artículos 
(Marcalyc) a diferentes formatos de lectura que están al alcance de todos nuestros lectores (Visor Redalyc, ePUB, Móvil, HTML).

\section{LOS IRTIICILOS NELL PRESEVTIE NÍNERO}

En este número 19 de IE Revista de Investigación Educativa de la REDIECH incluimos 17 artículos de diferentes regiones: dos del estado de Chihuahua, doce de entidades de la República Mexicana (Querétaro, Morelos, Ciudad de México, Guerrero, San Luis Potosí, Michoacán, Jalisco, Baja California y Nuevo León) y tres internacionales (Chile, Colombia y los Estados Unidos). Esta situación refleja un interés creciente hacia nuestra revista que se refleja en dos rubros: 1) considerable aumento de propuestas nacionales e internacionales, pues el porcentaje de aceptación del número fue de $31 \% ; y, 2$ ) participación de un equipo extenso de académicos que se involucraron tanto en el proceso editorial como en tareas de dictaminación (17 integrantes que realizaron las primeras lecturas de trabajos y 59 expertos evaluadores).

Las temáticas que abordan cada uno de los trabajos son diversas, encontrando el mayor número hacia aspectos de la educación superior. En el artículo "Alfabetización digital desde un enfoque instrumental, cognitivo y emocional en estudiantes de turismo usando Blackboard", Ana María Orozco Santa María, Ma. Teresa García Ramírez y Luis Alberto Cepeda Villasana abordan el uso de la tecnología en el aula, específicamente con estudiantes de turismo que cursan materias en línea en Blackboard. El propósito de la investigación es explorar la percepción de los estudiantes sobre su aprendizaje usando dicha herramienta. Entre los principales hallazgos que reportan son que los estudiantes perciben que aprenden mejor en cursos presenciales que en cursos en línea, lo cual sin duda aporta elementos para replantear la oferta 0 encontrar estrategias que ayuden a fortalecer los programas de educación virtual.

En el trabajo "Evaluación según diseño y aprendizaje de Google Classroom y Chamilo", Javier Tarango, Juan D. Machin-Mastromatteo y José Refugio Romo-González presentan una documento reflexivo, descriptivo y exploratorio relacionado con un instrumento de evaluación para sistemas de gestión del aprendizaje (SGA) que utilizaron para evaluar Chamilo y Google Classroom. Aunque los mismos autores reconocen ciertas limitaciones derivadas del proceso de investigación -pues solo reporta el punto de vista del profesor-, lo cierto es que puede contribuir en el desarrollo de mejores parámetros de evaluación aplicables a otros SGA.

Continúan en el tema de la educación superior el artículo de Martín Gerardo Martínez-Valdés y Luis Gibran Juárez-Hernández que se titula "Diseño y validación de un instrumento para evaluar la formación en sostenibilidad en estudiantes de educación superior", donde establecieron como propósito diseñar y validar un instrumento que delimite el grado de atención a los alumnos universitarios con respecto al tema de sostenibilidad, que sin duda es una aportación importante en el momento actual donde se está discutiendo el papel de la educación para apalear el problema del calentamiento global. Enseguida aparece "Educación para la empleabilidad: enfoque de la investigación educativa", de Eduardo Raúl Díaz Gómez, donde se aborda el papel de las universidades en la preparación de estudiantes para el empleo y desarrollo de competencias para la empleabilidad, a partir del análisis de la literatura que existe 
sobre el tema, pues actualmente son preocupantes los altos índices de desempleo entre jóvenes profesionistas en algunos países. Para cerrar el bloque temático, en el trabajo "Aprendizaje y práctica de la innovación en la universidad: actores, espacios y comunidades, Noé Abraham González-Nieto, Juan Manuel Fernández-Cárdenas y Cristina G. Reynaga-Peña analizan los datos obtenidos en un laboratorio de innovación de una universidad en el norte de México, donde estudiantes, profesores y colaboradores participan en proyectos interdisciplinarios para poner en práctica la innovación.

En el área del conocimiento matemático se presentan dos propuestas. Lilia Patricia Aké Tec nos trae el artículo titulado "Conocimiento matemático de maestros en formación sobre la simbología algebraica", que se ocupa de analizar la actividad matemática de los maestros en formación para la introducción y desarrollo del simbolismo algebraico. Por su parte, Javier García-García y Noe Bernandino-Silverio presentan "Conocimientos geométricos en la elaboración de un artefacto en una comunidad ñuu savi", que tiene como objetivo identificar las nociones geométricas presentes en el diseño y elaboración del güilile (un artefacto de uso extendido) en una comunidad ñuu savi. A partir de los resultados de la investigación se discuten algunas implicaciones para la enseñanza de contenidos matemáticos.

En los temas de política educativa, los trabajos nos llevan a diversos recorridos que explican las dinámicas educativas en México, Colombia y Chile. En el primero, Carlos Ramírez Silván y Silvia Patricia Aquino Zúñiga analizan "Los organismos internacionales y las políticas educativas de profesionalización docente de la educación normal en México", donde revisan la literatura sobre el papel que tienen los organismos internacionales en el establecimiento de las políticas de profesionalización docente de las escuelas normales. Ana María Guzmán Mora se ocupa de reflexionar en torno a la problemática de la instrucción en la escuela a partir del artículo "Tensiones y agencia en la escuela pública hoy", donde postula algunos elementos para la discusión sobre la configuración de la institución escolar en la actualidad, así como el papel del maestro en la construcción de esa institucionalidad. En el tercer trabajo del bloque, Catherine Evelyn Araya Pérez y José Alejandro González Campos presentan "Evaluación de la política pública en centros escolares suscritos a la Ley de Subvención Escolar Preferencial", donde analizan la política pública chilena que intenta reducir la brecha de resultados educativos entre escuelas municipales, particulares subvencionadas y privadas mediante la Ley de Subvención Escolar Preferencial que, aseguran -como resultado-, no ha tenido impacto para equiparar los resultados educativos.

En los temas que se refieren a la educación especial y vulnerabilidad social encontramos la aportación de Ismael García Cedillo, Bania Yarabí Hernández Hernández y Gabriela Silva Maceda titulada "Necesidades educativas especiales del estudiantado infantil y juvenil que vive con VIH", donde se proponen identificar si los niños y adolescentes que viven con VIH presentan necesidades educativas especiales y, de ser el caso, si están siendo debidamente atendidas en la escuela. Enseguida aparece "La desigualdad educativa en Michoacán medida a través del coeficiente de Gini", de Antonio Favila Tello y Plinio Hernández Barriga, quienes buscan hacer evidente la falta de diagnósticos a nivel estatal y municipal -en su estado- en el tema de la 
desigualdad educativa, que actualmente es uno de los principales problemas a nivel nacional. En el tercer trabajo del bloque, Daniela Almaraz Fermoso y Gabriela Coeto Cruzes presentan el trabajo "Habilidades sociales en niños de primaria", en el que muestran los resultados de un programa de habilidades sociales que se llevó a cabo en una primaria y que arrojó disminución en las conductas agresivas de los niños.

En el ámbito de la educación media superior aparecen dos trabajos. "Variables que discriminan a las víctimas y no víctimas de ciberacoso en adolescentes", de Remberto Castro Castañeda, Esperanza Vargas Jiménez y Claudia Gregoria Huerta Zúñiga", tiene como objetivo examinar las variables familiares, escolares, sociales e individuales que discriminan la cibervictimización en la adolescencia y a partir de ellas pueden predecir la incidencia de este fenómeno. La segunda aportación lleva por título "Intereses educativos, expectativas laborales y profesionales de los estudiantes de nivel medio superior en la Región Noroeste de Chihuahua: un análisis por género", de Ricardo López Salazar, Elizabeth Bautista Flores y Óscar Arturo Sánchez Carlos. Los autores exponen y analizan los resultados de una encuesta aplicada entre 2013 y 2014 a estudiantes que cursaron el último año del nivel medio superior en la región noroeste de Chihuahua, con el objeto de conocer los intereses educativos, las expectativas laborales y profesionales de acuerdo con el género.

En el rubro de la investigación educativa, Juan Carlos López García se ocupa de revisar "El congreso como objeto de estudio. Análisis de coautorías del Congreso de Investigación Educativa en Chihuahua, 2016 y 2018", referente al evento bianual que organiza la Red de Investigadores Educativos Chihuahua y en el que se pueden prever la configuración de comunidades académicas a partir de las relaciones entre autores (coautorías) y las relaciones interinstitucionales a que da lugar su afiliación laboral.

El número 17 cierra con el artículo "From the Zapatista narrative epistemology to the Academia Cuauhtli; Latinx-American students", de Raul Olmo Fregoso Bailón, quien describe cómo un grupo de estudiantes mexicanas de secundaria, en Guadalajara, México, escribió poemas con el objetivo de compartir sus reflexiones con los estudiantes que asisten a la Academia Cuauhtli, en Austin, Texas. A partir de la epistemología narrativa zapatista y el método de captar las percepciones epistemológicas de la poesía en educación, los poemas se agruparon en categorías para analizar el esfuerzo transnacional de la Academia Cuauhtli entre México y Estados Unidos.

Con esta entrega cerramos el segundo número de 2019, año en el que esta revista contó con el financiamiento del Conacyt para hacer posibles mejoras en la plataforma electrónica donde alojamos nuestros contenidos, por lo cual nos congratulamos de los avances que tiene este grupo de académicos entusiastas que se aglutinan alrededor de la Red de Investigadores Educativos Chihuahua, cuya presencia rebasa ya las fronteras del propio estado.

\section{ReFrenerills}

Aguado-López, E. y Becerril-García, A. (2019). R2020: modelo de publicación abierto, propiedad de la academia, sin fines de lucro, sustentable, con métricas responsables y no subordinado. Recuperado de https://www.redalyc.org/ 
Elsivier. (2019). Tipos de Open Access: diferencias entre la "vía verde" y la "vía dorada". Recuperado de: https://www.elsevier.com/es-es/connect/actualidadsanitaria/tipos-de-open-access-via-verde-y-la-via-dorada

Gobierno de la República. (2002, junio 5). Decreto por el que se expiden la Ley de Ciencia y Tecnología y la Ley Orgánica del Consejo Nacional de Ciencia y Tecnología. Diario Oficial de la Federación, (3), 65-89. Recuperado de http://www. dof.gob.mx/nota_to_imagen_fs.php?codnota $=727648 \&$ fecha $=05 / 06 / 2002 \&$ cod diario $=28651$

Gobierno de la República. (2019, mayo 15). Decreto por el que se reforman, adicionan y derogan diversas disposiciones de los artículos 30., 31 y 73 de la Constitución Política de los Estados Unidos Mexicanos, en materia educativa. Diario Oficial de la Federación, (13), 2-9. Recuperado de http://www.dof.gob.mx/nota to imagen fs.php? codnota $=5560457 \&$ fecha $=15 / 05 / 2019 \& \operatorname{cod}$ diario $=281840$

López Ruiz, M., Sañudo Guerra, L. y Maggi Yáñez, R. (2013). Investigaciones sobre la investigación educativa, 2002-2011. México: COMIE, ANUIES.

Ministerio de Economía y Empresa. (2017). Open Access: la vía verde, la vía dorada y la vía diamante. Recuperado de https://datos.gob.es/es/noticia/open-access-la-verde-ladorada-y-la-diamante

Redalyc. (2019). Principios y valores. Recuperado de https://www.redalyc.org/home.oa

Universidad de Salamanca. (2019). Acceso abierto: Plan S. Recuperado de https:// guiasbus.us.es/accesoabierto/plans 